\title{
Current Advances in Sustained Release Microneedles
}

\author{
Guangsheng Du ${ }^{1}$ Xun Sun ${ }^{1}$ \\ ${ }^{1}$ Key Laboratory of Drug-Targeting and Drug Delivery System of the \\ Education Ministry, Sichuan Engineering Laboratory for Plant- \\ Sourced Drug and Sichuan Research Center for Drug Precision \\ Industrial Technology, West China School of Pharmacy, Sichuan \\ University, Chengdu, PR China \\ Pharmaceut Fronts 2020;2:e11-e22.
}

\begin{abstract}
Address for correspondence Xun Sun, Key Laboratory of DrugTargeting and Drug Delivery System of the Education Ministry, Sichuan Engineering Laboratory for Plant-Sourced Drug and Sichuan Research Center for Drug Precision Industrial Technology, West China School of Pharmacy, Sichuan University, Chengdu, 610064, PR China (e-mail: sunxun@scu.edu.cn).
\end{abstract}

\begin{abstract}
Keywords

- sustained release

- polymeric microneedles

- drug delivery

- vaccines

Microneedles have been extensively investigated for intradermal delivery of drugs and vaccines due to advantages including high skin delivery efficiency, improved patient compliance, and potential for self-administration. However, traditional microneedles cannot regulate the release kinetics of payloads, limiting therapeutic utility of the biotherapeutics. Recently, several types of microneedles with sustained release properties, including slow-dissolving microneedles made of hydrophilic polymers, degradable microneedles made of hydrophobic polymers, and bioresponsive microneedles made of bioresponsive polymers, have been developed and investigated for intradermal delivery of the biotherapeutics, aiming for improving their therapeutic potency, reducing side effects and administration frequency, and further improving patient compliance. In this review, we introduced different types of microneedles that have been designed for sustained release of the payloads, summarized various applications of these microneedles, and discussed the future prospects of this technology.
\end{abstract}

\section{Introduction}

Microneedles are microscale structures that are designed to overcome the skin barrier for successful intradermal delivery of drugs and vaccines. They generally have a length ranging from $200 \mu \mathrm{m}$ to $1 \mathrm{~mm}$ and therefore can avoid stimulating nerve endings when applied into the skin. ${ }^{1-4}$ Compared with traditional injections with hypodermic needles, microneedle-mediated intradermal delivery of biotherapeutics have several advantages, including improved patient compliance, potential for self-administration, avoidance of needle stick injury, and infection risk due to reuse of needles. ${ }^{5}$ Microneedles can also improve the stability of loaded biotherapeutics and potentially decrease side effects associated to systemic administration. ${ }^{6-11}$

Microneedles have been classified into coated, hollow, and polymer-based dissolving microneedles. The fabrication methods, various features, and applications of these microneedles have been extensively summarized and discussed in several good review articles. ${ }^{1,2,12-14}$ Among the different types of the microneedles, dissolving microneedles which are made of fast water-soluble polymers have received particular attention as they do not result in any hazardous sharp waste after application. ${ }^{15-19}$ They can normally dissolve within minutes and release the loaded biotherapeutics as the matrix dissolves. ${ }^{18,20-22}$ In the past two decades, dissolving microneedles have been fabricated by using various types of biocompatible and water-soluble matrix, including hyaluronic acid (HA), ${ }^{23,24}$ polyvinylpyrrolidone (PVP), ${ }^{25,26}$ sucrose, ${ }^{27,28}$ gelatin, ${ }^{29}$ etc. Microneedles made of these polymers have shown strong mechanical strength for skin piercing and excellent biocompatibility and safety for delivery of substances from small-molecule chemical drugs to biomacromolecule protein therapeutics. ${ }^{30-34}$ However, received

November 14, 2019

accepted

December 4, 2019
DOI https://doi.org/

$10.1055 / \mathrm{s}-0040-1701435$

ISSN 2628-5088. (c) 2020 Georg Thieme Verlag KG Stuttgart . New York
License terms

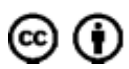


most of the investigated microneedles cannot regulate the release kinetics of the encapsulated cargos due to the instant dissolution properties of the matrix. As a result, the microneedle-delivered drugs may have a short half-life and unordered distribution, which may limit their potency and cause unwanted side effects. ${ }^{35}$

To exploit the potential of polymeric microneedles for modulation of release properties and further improve therapeutic potency of the payloads, researchers started to design and investigate microneedles with sustained release properties. $^{36}$ Instead of rapidly dissolving and releasing the drugs after insertion, sustained release microneedles can keep the drug inside the matrix and release the payloads in a certain manner based on the dissolution or degradation properties of the polymer matrix. ${ }^{36,37}$ The hypothesis is that the sustained release of drugs and vaccines from the microneedles could help to improve therapeutic efficacy, decrease side effects, and reduce administration frequency. To this end, several types of microneedles have been developed, including slow-dissolving microneedles made of hydrophilic polymers, degradable microneedles made of hydrophobic polymers, and bioresponsive microneedles made of bioresponsive polymers. ${ }^{37}$ In the case of slow-dissolving microneedles, the release rate of the payloads is determined by the dissolution of the polymer after the insertion into skin. In degradable microneedles, the drug is delivered through passive diffusion or degradation of the matrix. In bioresponsive microneedles, bioresponsive polymers and micro- or nanoparticles which are sensitive to physiological signal are utilized for drug delivery in a bioresponsive manner ${ }^{37}$ (-Table 1).

Although the research of these sustained release microneedles is in a relatively early stage, they have been used for delivery of various types of drugs and vaccines, including small-molecule chemical drugs, antibodies, protein therapeutics, and vaccines. These microneedles have been investigated for immune modulation, cancer therapy, diabetes treatment, etc. The results have shown that the sustained release of drug from the microneedles can improve therapeutic potency of the cargos and sustained release microneedles have potential to be used as a patient-friendly substitute for conventional sustained release methods. In this review, we introduced the representative types of the microneedles that have been designed for sustained release of drugs and vaccines, summarized different applications of these microneedles, and discussed the future perspective of this technology.

\section{Representative Microneedle Types with Sustained Release Properties}

\section{Slow-Dissolving Microneedles Made of Hydrophilic Polymers}

To extend the release period of drugs of dissolving microneedles, hydrophilic polymers with a slow dissolution rate in aqueous media were used. The polymers that have been studied for this type of microneedles are chitosan and polyvinyl alcohol (PVA; - Table 2) ${ }^{38-40}$ When selecting these polymers, the parameters that need to be carefully considered are water solubility, mechanical strength, and compatibility to drugs and vaccines. Although several new methods, including drawing lithography, ${ }^{41}$ soft lithography, ${ }^{42}$ and droplet-born air blowing, ${ }^{43}$ have been developed for fabrication of polymeric microneedles, the mostly used method for preparing sustained release microneedles is the aqueousbased micromolding method. ${ }^{2,12,32}$

To fabricate slow-dissolving microneedles, one challenge is to dissolve the polymer in aqueous solution and obtain a relatively high concentration, which is needed for micromolding. Researchers have utilized high temperature and acidic $\mathrm{pH}$ for helping in dissolving the polymers with a high concentration. For example, to prepare a matrix formulation of PVA/PVP-based microneedles, Leonard et al first dissolved PVA in water to obtain a concentration of $0.67 \mathrm{~g} / \mathrm{mL}$ by heating at $90^{\circ} \mathrm{C} .{ }^{40}$ After adding PVP, the polymer mixtures were further incubated at $60^{\circ} \mathrm{C}$ for 5 to 6 hours before use. In another study, chitosan was used to prepare a microneedle patch for sustained delivery of a model antigen ovalbumin (OVA). A $2 \%$ chitosan solution was first prepared by adding $2 \%$ (w/v) acetic acid. Next, excess acetic acid was removed by dialysis and excess water was removed by evaporation to obtain a $10 \%$ chitosan gel. The prepared chitosan microneedles successfully penetrated the skin, leaving the chitosan tip embedded for sustained release of OVA for over 14 days (-Fig. 1). ${ }^{38}$ These high temperature and acidic conditions, however, may impact the structure and functionality of the loaded biotherapeutics, especially for protein and gene drugs which are sensitive to environmental conditions. $^{44-47}$

Another strategy that can enable dissolving microneedles to release the payloads in a controlled manner is to combine nano- and microparticle technologies. ${ }^{48,49}$ Vora et al fabricated PVP-based microneedles loaded with a mixture of poly (lactic-co-glycolic acid) (PLGA) nanoparticles and microparticles. ${ }^{48}$ The developed system showed good mechanical and

Table 1 Different types of sustained release microneedles and their drug release mechanism

\begin{tabular}{|l|l|l|}
\hline Microneedle type & Polymer matrix & Drug release mechanism \\
\hline $\begin{array}{l}\text { Slow-dissolving microneedles made of } \\
\text { hydrophilic polymers }\end{array}$ & Slowly water-dissolving polymers & $\begin{array}{l}\text { Released together with the dissolution } \\
\text { of matrix }\end{array}$ \\
\hline $\begin{array}{l}\text { Degradable microneedles made of } \\
\text { hydrophobic polymers }\end{array}$ & $\begin{array}{l}\text { Non-water soluble and degradable } \\
\text { polymers }\end{array}$ & $\begin{array}{l}\text { Released through passive diffusion or } \\
\text { degradation of polymer }\end{array}$ \\
\hline $\begin{array}{l}\text { Bioresponsive microneedles made of } \\
\text { bioresponsive polymers }\end{array}$ & $\begin{array}{l}\text { Biodegradable and bioresponsive } \\
\text { polymers }\end{array}$ & $\begin{array}{l}\text { Released in responding to physiological } \\
\text { signals }\end{array}$ \\
\hline
\end{tabular}


Table 2 Representative microneedle types for sustained delivery of biotherapeutics

\begin{tabular}{|c|c|c|c|}
\hline Microneedle type & Polymer matrix & Delivered biotherapeutics & Ref. \\
\hline \multirow{5}{*}{$\begin{array}{l}\text { Slow-dissolving microneedles made } \\
\text { of hydrophilic polymers }\end{array}$} & Chitosan & OVA & 38 \\
\hline & Chitosan & Calcein, BSA & 39 \\
\hline & PVA & $\begin{array}{l}\text { Sulforhodamine B, inactivated } \\
\text { influenza virus }\end{array}$ & 40 \\
\hline & PVP, PLGA & Vitamin D3 & 48 \\
\hline & PVA, PLGA & Alexa 488, Cy5 & 49 \\
\hline \multirow{16}{*}{$\begin{array}{l}\text { Degradable microneedles made of } \\
\text { hydrophobic polymers }\end{array}$} & PLGA & Calcein, BSA & 50 \\
\hline & PLGA & $\begin{array}{l}\text { Sulforhodamine } B \text {, inactivated } \\
\text { influenza virus }\end{array}$ & 40 \\
\hline & PLA & Methotrexate & 51 \\
\hline & PLGA & Rhodamine & 52 \\
\hline & PLGA & OVA & 53 \\
\hline & PLGA, PLA & Levonorgestrel & 54 \\
\hline & PLGA & OVA & 62 \\
\hline & $\begin{array}{l}\text { Crystallized silk } \\
\text { fibroin }\end{array}$ & OVA & 56 \\
\hline & Crosslinked MeHA & Anti-PD-1 & 57 \\
\hline & $\begin{array}{l}\text { Genipin-crosslinked } \\
\text { gelatin }\end{array}$ & Insulin & 58 \\
\hline & $\begin{array}{l}\text { Crystallized silk } \\
\text { fibroin }\end{array}$ & Antibiotic & 60 \\
\hline & PS-PAA & Insulin & 113 \\
\hline & Crosslinked MeHA & Tumor lysate & 61 \\
\hline & Crosslinked MeHA & Insulin & 63 \\
\hline & Crosslinked MeHA & Insulin & 64 \\
\hline & Crosslinked MeHA & Checkpoint inhibitors & 65 \\
\hline \multirow{4}{*}{$\begin{array}{l}\text { Bioresponsive microneedles made of } \\
\text { bioresponsive polymers }\end{array}$} & Crosslinked MeHA & Insulin & 79 \\
\hline & Crosslinked PVA & Insulin & 66 \\
\hline & Crosslinked MeHA & Insulin & 80 \\
\hline & Crosslinked alginate & Exendin-4 & 67 \\
\hline
\end{tabular}

Abbreviations: BSA, bovine serum albumin; OVA, ovalbumin; PLA, polylactic acid; PLGA, poly(lactic-co-glycolic) acid; PVA, polyvinyl alcohol; PVP, polyvinylpyrrolidone.

insertion profiles, and can continuously release a model drug for over 5 days. In another study, Ke et al developed PVP based dissolving microneedles loaded with $\mathrm{pH}$-sensitive PLGA microparticles for multidrug release in sequence. In the first step, the microneedles quickly dissolved and released the free drug as well as another drug encapsulated in PLGA microparticles. In the subsequent step, the delivered microparticles disassembled due to the acidic $\mathrm{pH}$ of the skin and released the second drug. This system has potential for use of some clinical applications in which drugs need to be administered in sequence. ${ }^{49}$ However, in the studies mentioned above, the authors did not investigate the application of the developed microneedles in real disease models. Additionally, the relatively low loading capacity of the polymeric nanoparticles may limit the overall drug loading capacity of the microneedles.

\section{Degradable Microneedles Made of Hydrophobic Polymers}

Except utilizing dissolving microneedles for sustained drug release, researchers also designed degradable microneedles by using nonsoluble and hydrophobic polymers for sustained release of drugs. The polymers that have been used are polylactic acid and PLGA, which have good biocompatibility and biodegradability (- Table 2). ${ }^{40,50-54}$ Degradable microneedles made of these polymers have shown sufficient mechanical strength for skin insertion and a slow degradation rate for sustained release of the payloads. Hydrophobic drugs can be directly dissolved and mixed with the polymer in organic solution for drug loading during the micromolding process. ${ }^{51}$ In most of the cases, the polymers need to be melted at a high temperature above $135^{\circ} \mathrm{C}$ in order to fill the microcavities of the molds. ${ }^{50,53}$ The high temperature and 
A
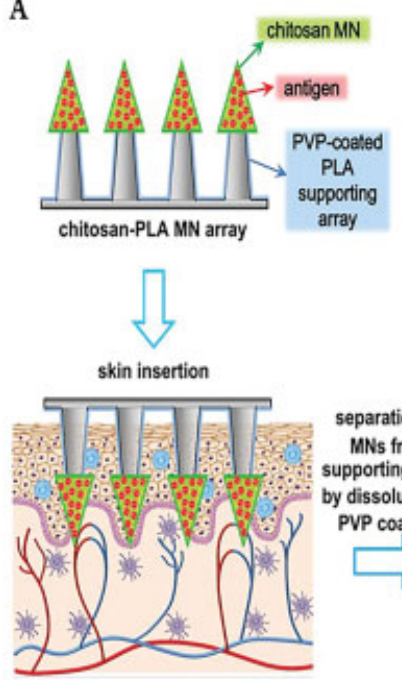

sustained release of antigen

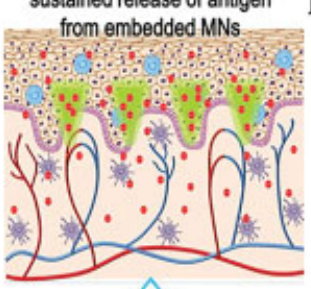

removal of supporting array
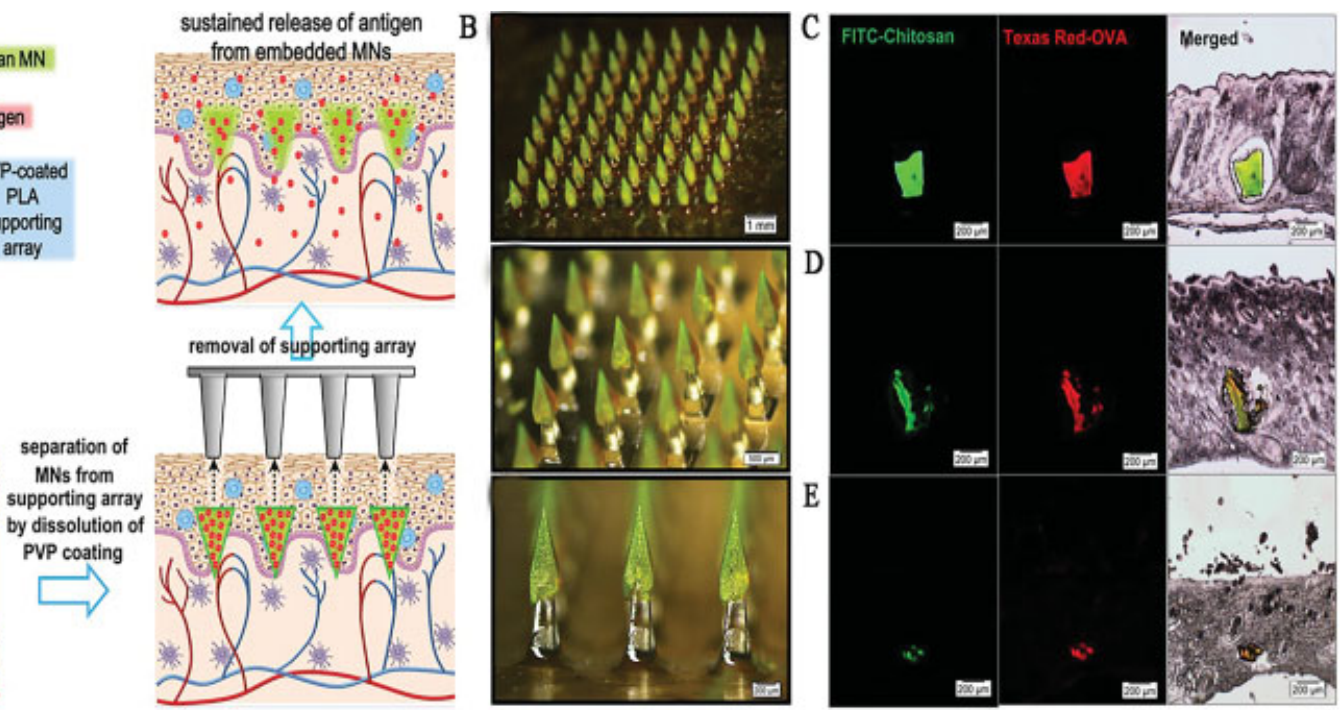

Fig. 1 Chitosan-based microneedles for sustained release of OVA. (A) Schematic illustration of the fabrication process. (B) Bright-field images of the microneedles. (C-E) Confocal images of the insertion sites after 1 day (C), 1 week (D), and 2 weeks (E). OVA, ovalbumin. (Adapted with permission from Chen et al $2013^{38}$.)

organic solvent used in this method could cause the loss of drug efficacy. ${ }^{55}$ The loading of hydrophilic drugs can be a challenge due to the immiscible nature of the drug and microneedle matrix. In some studies, hydrophilic drugs were first encapsulated into nano- or microparticles, and the particles were incorporated into the microneedles in the next step. ${ }^{50,52}$

Preparing degradable microneedles by postprocessing is an interesting alternative method that has been developed recently for overcoming the drawbacks mentioned above. ${ }^{56-65}$ This approach combines the advantages of easiness for drug loading of dissolving microneedles during the preparation process and sustained release properties of biodegradable polymers. To prepare the microneedles, chemically modified water-soluble polymers were first used for the loading of drugs and vaccines. After molding, the fabricated microneedles were crosslinked or crystallized by chemical or physical treatments including UV exposure, organic solvent treatment, etc. After the postprocessing, the polymers became non-water soluble and thereby could slowly release the drug during degradation. Studies have shown that the crosslinked or crystallized microneedles can significantly improve the stiffness of microneedles and extend the release time of the payloads. The matrices used for this type of microneedles include crosslinked methacrylated hyaluronic acid (MeHA), ${ }^{57,61,63-65}$ crosslinked PVA, 66 genipin-crosslinked gelatin, ${ }^{58}$ crystallized silk fibroin, ${ }^{59,60}$ and crosslinked alginate. ${ }^{67}$

Researchers from Gu et al's group fabricated crosslinked MeHA microneedles and applied them for the delivery of checkpoint inhibitors, tumor lysates, and insulin (-Fig. 2). ${ }^{57,61,63-65}$ HA was first modified with a methyacrylamide group and filled into micromolds together with methylenebisacrylamide and a photoinitiator for microneedle fabrication. After UV exposure, the polymers form a water-insoluble network entrapping the drug, allowing for sustained release of drug for over several days. ${ }^{57,61,65}$ The methyacrylation rate of $\mathrm{HA}$ and the exposure time to UV light have been shown to influence the mechanical strength of microneedles and release properties of the payloads. Similarly, they modified PVA with the methyacrylamide group and fabricated crosslinked PVA microneedles for sustained release of payloads. ${ }^{66}$

Silk fibroin can be used for microneedle preparation due to their excellent mechanical properties, biocompatibility, and biodegradability ${ }^{68,69}$ However, microneedles made of silk fibroin without any processing cannot control the release rate of the payloads. ${ }^{70}$ Recent studies showed that the degradation rate of silk fibroin and the diffusion rate of the payloads can be controlled by postprocessing the microneedles with methanol or high humidity exposure, by changing the secondary structure of the silk fibroin. ${ }^{71-73}$ Tsioris et al modified silk fibroin with water vapor annealing for modulation of the degradation properties of silk fibroin. They showed that the processed silk fibroin had higher content of $\beta$ sheet secondary structure, and the drug release rate was decreased by 5.6 -fold compared with nonmodified microneedles. ${ }^{60}$ Jiyong et al. treated silk-fibroin-based microneedles with methanol, and found that the exposure time to methanol can be used to control the drug release rate. ${ }^{59}$

\section{Bioresponsive Microneedles Made of Bioresponsive Polymers}

The slow-dissolving microneedles and degradable microneedles can deliver the loaded drugs and vaccines in a sustained manner, which is predetermined by the dissolution and biodegradation rate of the polymers. Bioresponsive microneedles, in contrast, can respond to the physiological signal and release the payloads smartly according to the change of the physiological signals..$^{74,75}$ This character of the microneedles can be achieved via the loading of drugs in bioresponsive polymers or secondary encapsulation of drugs in physiological signalsensitive micro- or nanoparticles. ${ }^{76-78}$ The microneedles keep 
A
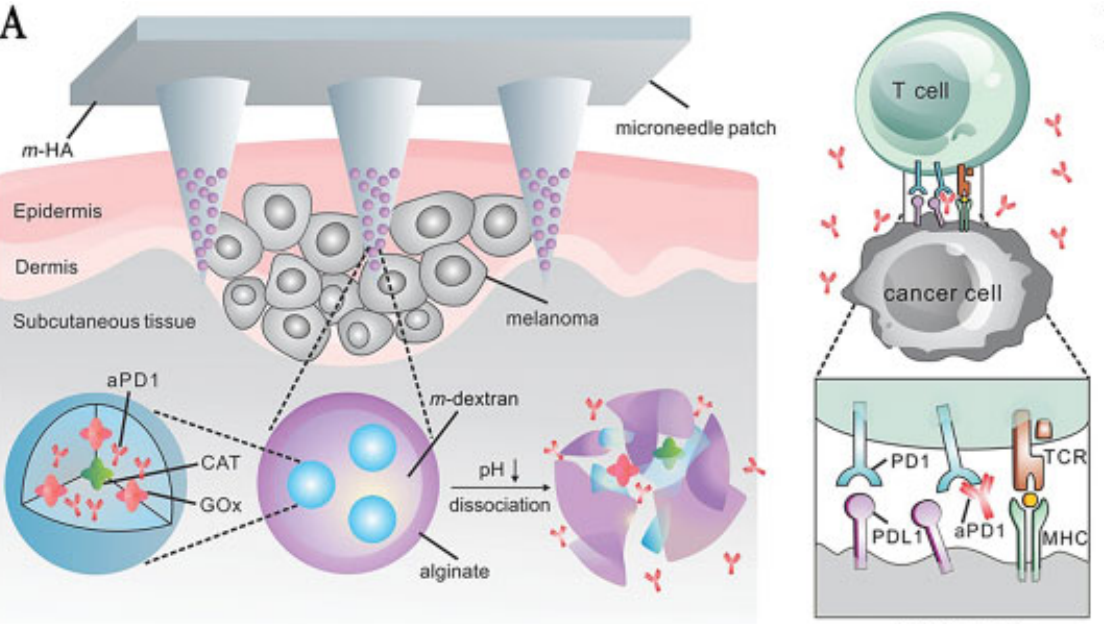

PD1 blockade
B

$\mathrm{C}$
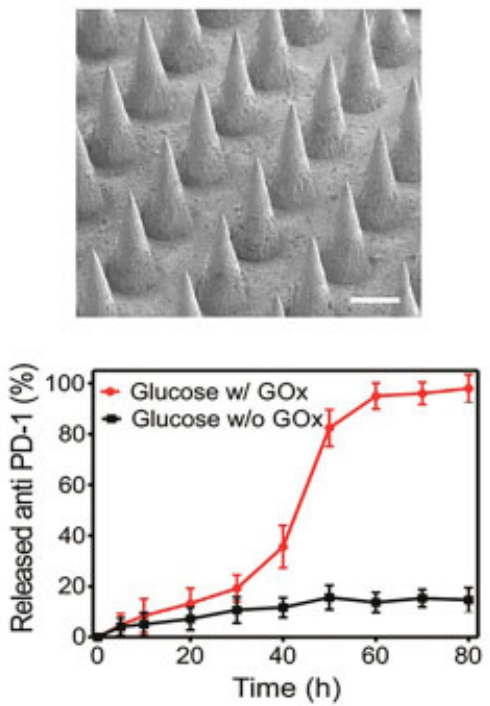

$\mathrm{E}$

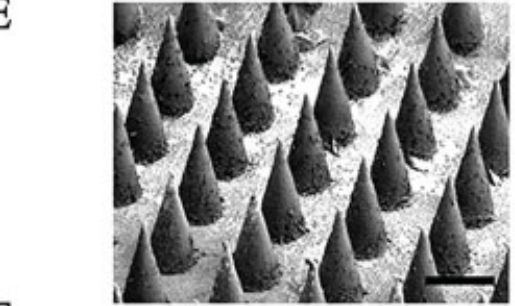

$\mathrm{F}$

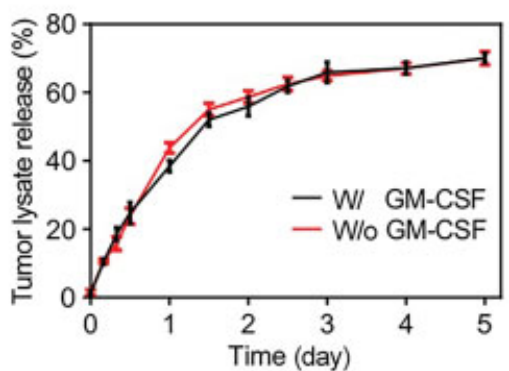

Fig. 2 Crosslinked MeHA microneedles used for delivery of checkpoint inhibitors and tumor lysates. (A) Schematic of anti-PD-1 delivery by crosslinked MeHA microneedles. (B) Scanning electron microscopy (SEM) image of anti-PD-1-loaded microneedles. (C) In vitro release of anti-PD1 from crosslinked MeHA microneedles. (D) Schematic of sustained delivery of tumor lysates by crosslinked MeHA microneedles. (E) SEM image of tumor-lysate-loaded microneedles. (F) In vitro release of tumor lysates from the microneedles. MeHA, methacrylated hyaluronic acid. (Adapted with permission from Wang et al $2016^{57}$ and Ye et al $2017^{61}$.)

the drug in the matrix, while the particles can respond to the physiological signals and release the drug in a sustained manner.

The bioresponsive microneedles that have been reported in the literature are mainly glucose-responsive ones (-Table 2). ${ }^{66,79,80}$ In this system, the glucose oxidase is an essential component which is normally encapsulated inside of nanoparticles embedded in the microneedle matrix. The glucose oxidase can catalyze the oxidation of high concentration of blood glucose and produce $\mathrm{H}_{2} \mathrm{O}_{2}$, which in turn stimulates the release of insulin from $\mathrm{H}^{+}$- or hypoxia-sensitive nanoparticles. This process finally can lead to a closed loop and regulate the insulin delivery in a bioresponsive manner. For example, researchers fabricated bioresponsive microneedles loaded with $\mathrm{pH}$-sensitive and insulin-loaded nanoparticles. The $\mathrm{H}_{2} \mathrm{O}_{2}$ produced from the oxidation of glucose by glucose oxidase successfully triggered the release of insulin from the nanoparticles. ${ }^{66,80}$ In these two studies, the outer layer of microneedles was loaded with a catalyzing enzyme for scavenging excess $\mathrm{H}_{2} \mathrm{O}_{2}$ to protect normal tissues from injury caused by oxidative stress. ${ }^{66}$ Similarly, Chen et al fabricated crosslinked alginate microneedles loaded with dual-mineralized nanoparticles for encapsulation of exendin- 4 and glucose oxidase separately. The $\mathrm{H}^{+}$produced by enzyme oxidation of blood glucose stimulated the disassembling of mineralized nanoparticles, leading to bioresponsive release of insulin. ${ }^{79}$

\section{Application of Microneedles with Sustained Delivery Properties}

Fast dissolving microneedles have the limitation that the drug release patterns cannot be precisely controlled. Sustained 
release microneedles open up new therapeutic utility for applications which require constant delivery of the drugs. The microneedles with sustained delivery properties can potentially be used to improve therapeutic potency, decrease administration frequency, and reduce side effects. The applications that have been investigated so far are immune modulation, cancer therapy, and diabetes treatment.

\section{Immune Modulation}

The skin harbors abundance of antigen-presenting cells, including Langerhans cells in the epidermis and dendritic cells in the dermis, making the skin an important site for vaccination. ${ }^{81-85}$ It has been shown that intradermal vaccination can result in stronger immune responses as compared with subcutaneous and intramuscular vaccinations. ${ }^{86-89}$ Furthermore, the vaccination using microneedles can avoid pain sensation and significantly reduce the stress of recipients as compared with injection by traditional hypodermic needles, which is especially important for vaccination of young children. ${ }^{90,91}$

Sustained release microneedles can be used to further improve the immunogenicity of the loaded antigen. During natural infection with microorganism, the pathogens replicate typically over several weeks and therefore continuously expose the antigen to the immune system. As a result, the induced immune response is normally strong. ${ }^{92,93}$ Previous studies have also shown that the sustained delivery of antigens and adjuvants from nanoparticles can potentially increase the immune responses. ${ }^{94-97}$ Microneedles with a sustained release behavior can be used as a painless and patient-friendly alternative mimicking this natural infection for improving the immunogenicity of antigens. ${ }^{53,56}$ One study showed that by using silk-fibroin-fabricated microneedles for sustained delivery of loaded OVA, the elicited antibody titers was 10 -fold higher than that elicited by traditional hypodermic-needleinjected antigens ( - Fig. 3). ${ }^{56}$ The same group also investigated PLGA-based microneedles for intradermal delivery of OVA and found that the sustained delivery of OVA significantly increased T-cell responses although did not improve antibody titers. ${ }^{53}$

Another disadvantage of traditional hypodermic-needlemediated immunization is that normally multiple injections are needed to stimulate robust immune responses. ${ }^{98}$ As a result, the recipients may fail to follow the entire program for successful immunization. On the other hand, these disadvantages bring several burdens for caregivers, especially during large vaccination campaigns. The use of sustained release microneedles for vaccination thereby has potential to significantly improve the vaccine coverage and reduce the work of caregivers. ${ }^{99}$ To the best of the authors' knowledge, however, there are no studies reported to date investigating the potential of sustained release microneedles for reducing immunization frequency.

\section{Cancer}

Recently, cancer treatment with immune therapy by harnessing the immune system has achieved great progress, including immunotherapy by using checkpoint inhibitors and cancer vaccines. These strategies aim at stimulating the immune system or remodeling the tumor microenviron- ment, and finally eliciting potent $\mathrm{CD}^{+} \mathrm{T}$-cell responses for clearance of cancerous cells. ${ }^{100-102}$ Immunotherapy strategies by using anti-PD- 1 and anti-CTLA- 4 have achieved encouraging results in clinical trials and are now revolutionizing cancer treatments. ${ }^{103}$ However, the response rate is relatively low and cancer-type-dependent. ${ }^{104,105}$ Researchers have tried to combine different immune reagents and treatment strategies to achieve synergetic antitumor efficacy. Microneedles with sustained release properties provide a good platform for this purpose for remodeling immune microenvironments and stimulating the immune system.

Wang et al developed a self-degradable microneedle patch composed of anti-PD-1 and glucose oxidase that are loaded in $\mathrm{pH}$-sensitive dextran nanoparticles. After insertion into the skin, the $\mathrm{pH}$ was decreased due to the oxidation of glucose and production of $\mathrm{H}^{+}$, which triggered the degradation of the nanoparticles for sustained release of anti-PD-1. This selfdegradable microneedles induced stronger immune responses and antitumor potency compared with microneedles without self-degradation property. ${ }^{57}$ Ye et al loaded B16F10 tumor lysates and photothermal reagents in crosslinked MeHA microneedles. The results showed that the developed microneedles could continuously release loaded tumor lysates under exposure to laser light. This combined strategy elicited potent antitumor effects and significantly increased the survival rate of tumor-bearing mice. ${ }^{61}$ They also developed crosslinked MeHA microneedles for co-loading of anti-PD-1 and IDO (indoleamine 2,3-dioxygenase) inhibitor 1-MT. The sustained release of these two inhibitors successfully enhanced the retention of the checkpoint inhibitors in the tumor microenvironment. This system finally demonstrated a synergistic treatment effect against B16F10 mouse melanoma. ${ }^{65}$

\section{Diabetes}

For diabetes, subcutaneous injection and pump-mediated infusion of insulin are the main treatment strategies. ${ }^{106}$ These delivery strategies, however, could cause complications including acute pain and inflammation due to frequent administrations for maintaining an appropriate blood concentration of insulin. Under this context, researchers have investigated the microneedle system for noninvasive and convenient intradermal delivery of insulin. ${ }^{38,107-109}$ However, there are two limitations of these microneedle systems. First, the investigated microneedle systems cannot control the delivery kinetics of insulin and therefore have a short lasting time for glucose control. Second, the delivered dose of insulin is predetermined and there is a risk that the administrated insulin may exceed the amount that is needed, causing hypoglycemia which can be severe and lethal. ${ }^{110-112}$ To extend the release time of insulin from the microneedle system, researchers utilized biodegradable microneedles for sustained release of insulin. ${ }^{58,113}$ These systems have shown to be able to extend the release of insulin and maintain the glucose level in a stable range within 10 to 12 hours. For instance, Chen et al fabricated genipin-crosslinked gelatin microneedles for sustained delivery of insulin. They observed that the crosslinking degree of gelatin could affect the mechanical strength and release profile of the microneedles. The microneedles with a higher crosslinking degree 
A

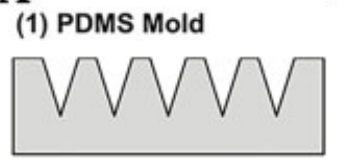

(2) Silk Addition

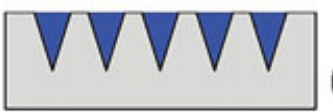

(3) Silk Drying $\pm \mathrm{MeOH}$

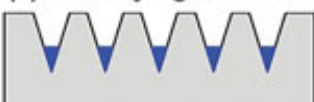

(4) PAA Addition

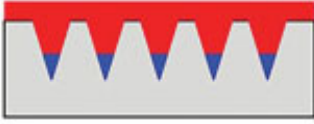

(5) PAA Drying/Removal

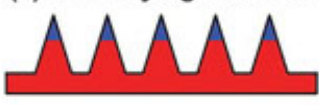

B
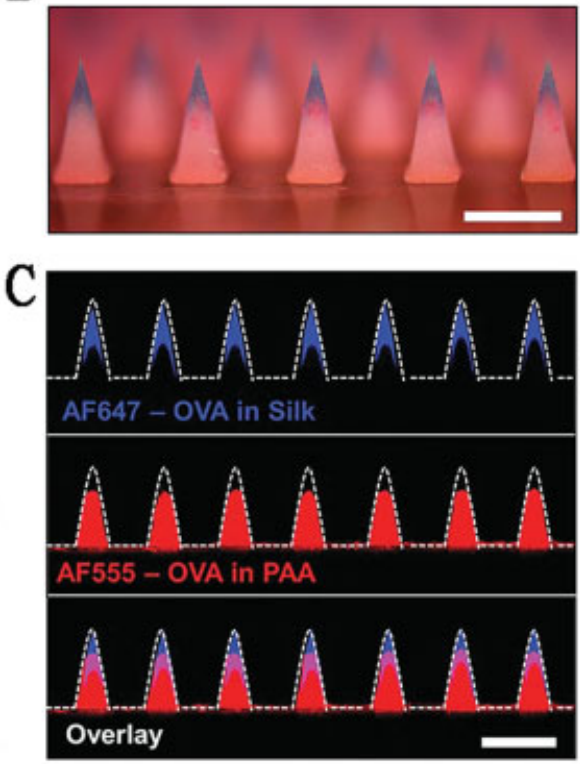

D

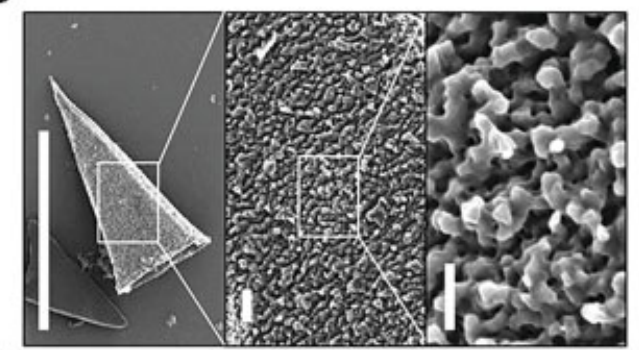

$\mathrm{E}$

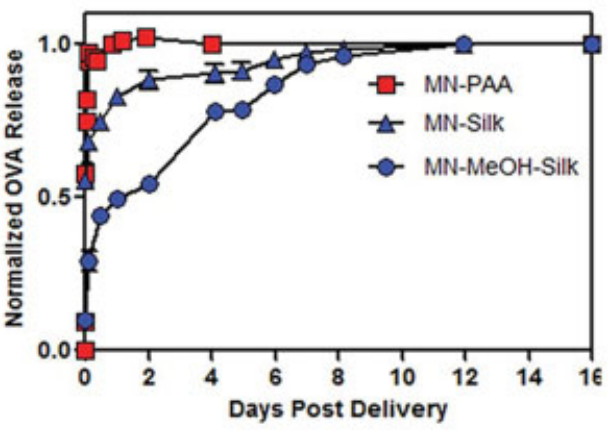

F
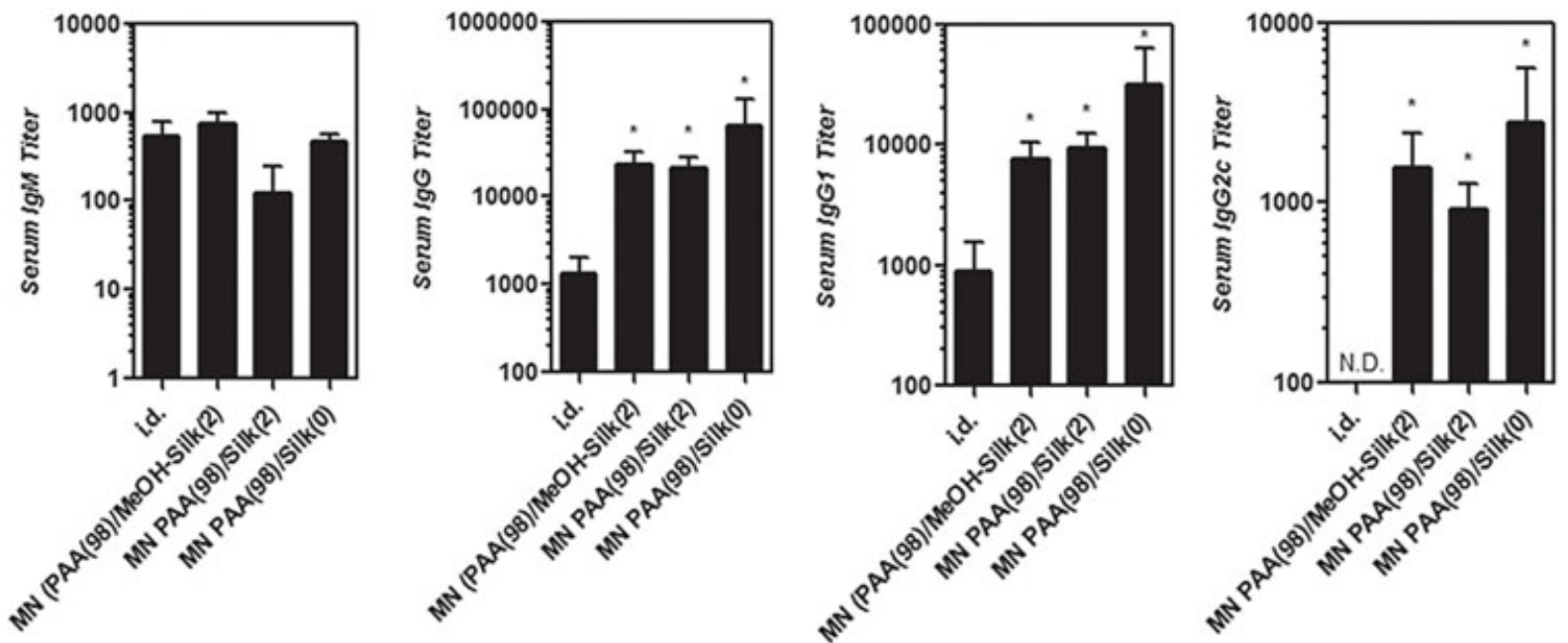

Fig. 3 Crystallized silk fibroin microneedles used for modulation of immune response. (A) Schematic of fabrication of microneedles. (B) Optical images of OVA-loaded microneedles. (C) Confocal images of OVA-loaded microneedles. (D) SEM image of a single microneedle. (E) In vitro release of OVA from the microneedles. (F) Antibody immune responses after immunization with the sustained release microneedles in mice. OVA, ovalbumin; SEM, scanning electron microscopy. (Adapted with permission from DeMuth et al $2014^{56}$.)

could more prolong the release of insulin and improve the therapeutic effectiveness of insulin as compared with traditional subcutaneous injection. ${ }^{58}$ Seong et al fabricated microneedles with a bullet shape made of swellable polystyreneblock-poly(acrylic acid) (PS-PAA) for intradermal delivery of insulin. They showed that after inserting microneedles into the skin, the loaded insulin could be delivered through passive diffusion due to the swelling of the tip matrix. The in vitro release study showed that the microneedles had released around $60 \%$ of the payloads for over 12 hours. In a rat diabetes animal model, the microneedle administration had led to a gradual decrease of blood glucose levels for over 8 hours. ${ }^{113}$

One risk of traditional insulin injection is that excess insulin may cause hypoglycemia. Therefore, a new "smart" system for insulin delivery is urgently needed that can deliver desirable amounts of insulin when the blood glucose level is high while maintaining the basal release rate of insulin when the blood glucose level is normal. To this end, researchers developed bioresponsive microneedles that can precisely release the loaded insulin in response to the blood glucose level, as discussed in the section of "bioresponsive microneedles." This system could help create a closed loop for insulin delivery, avoiding the risk of excessive delivery of the drug. Zhang et al developed crosslinked MeHA bioresponsive microneedles for entrapment of insulin and glucose oxidase. The results showed that the developed system can effectively regulate the blood glucose level within a normal range for around 12 hours after administration of one microneedle patch on a diabetic mice model (-Fig. 4). ${ }^{66,80}$ Wang et al also investigated the multiple microneedle administration and monitored the blood glucose 
A

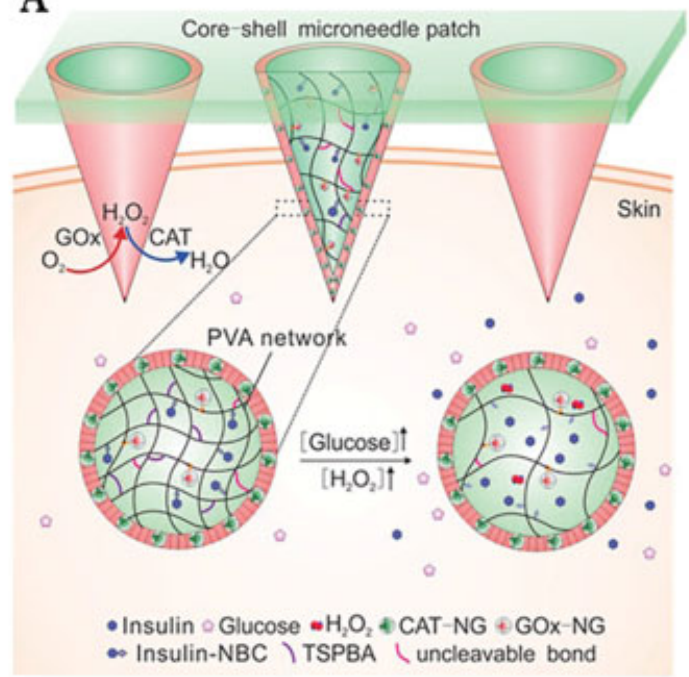

B

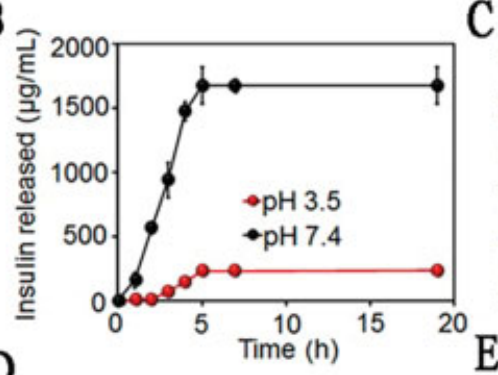

D

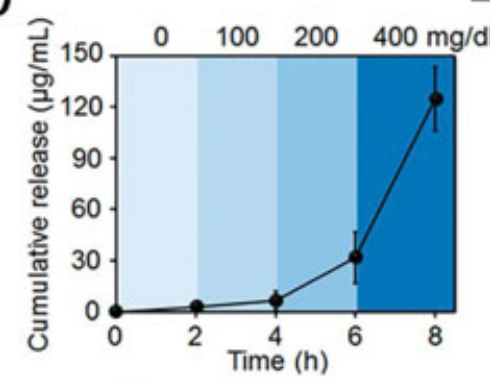

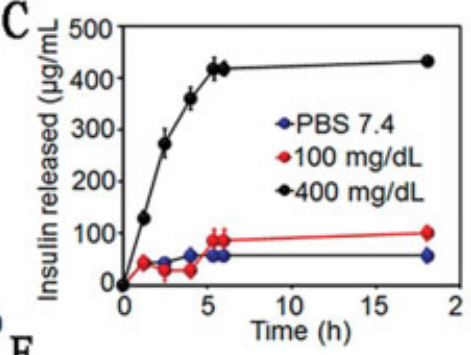

E

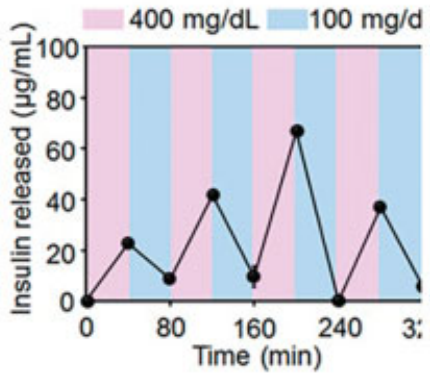

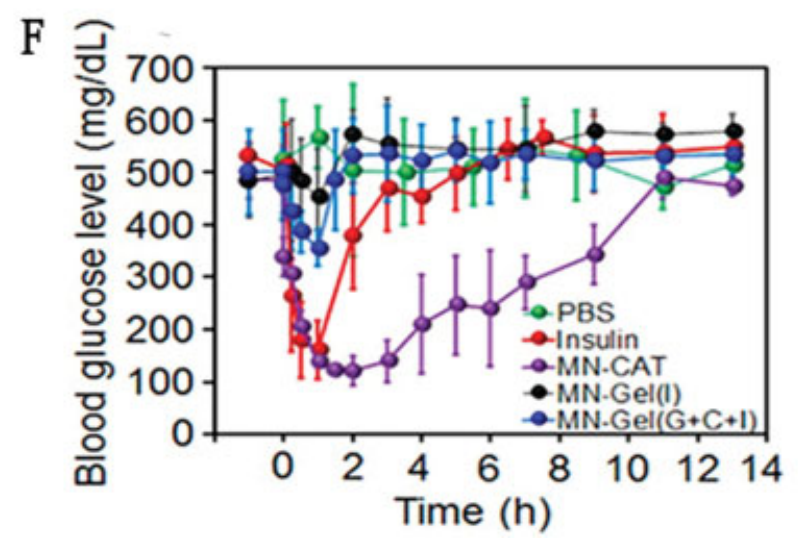

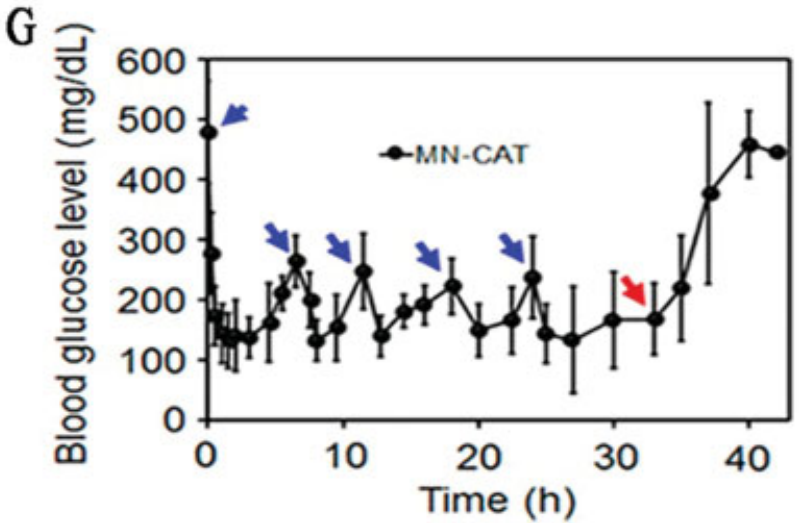

Fig. 4 Crosslinked PVA-based bioresponsive microneedles for regulation of blood glucose level. (A) Schematic of fabrication of microneedles. (B) The release of insulin from crosslinked PVA gel at different pH values. (C) The release of insulin from crosslinked PVA gel at different glucose concentrations. (D) The release of insulin with different concentrations of glucose. (E) Self-regulated release of insulin in response to glucose level. (F) The blood glucose level for over 14 hours after application of one microneedle patch on diabetes mice model. (G) The blood glucose level for over 40 hours after application of multiple microneedle patches on diabetes mice model. PVA, polyvinyl alcohol. (Adapted with permission from Ye et al $2016^{66}$.)

level for 40 hours. They found that within this time period, the blood glucose level was regulated to a narrow range between 100 and $250 \mathrm{mg} / \mathrm{dL}$ without any hypoglycemia observed. ${ }^{66}$

\section{Other Applications}

The sustained release microneedles were also investigated for other applications, including ocular disease, ${ }^{51}$ contraception, ${ }^{54}$ and antibacterial. ${ }^{60}$ In these studies, the microneedles were used to maintain and achieve sufficient therapeutical levels of the payloads with less administration frequency, aiming for better patient acceptance, improved safety, and stronger therapeutic efficacy.

\section{Conclusion and Perspectives}

Sustained release microneedles have shown superior delivery efficacy as compared with fast-dissolving microneedles. However, there are still several aspects that need to be further investigated or optimized. First, the small drug loading capacity of the microneedles due to their small size and volume is a limiting factor for their clinical translation. Researchers have utilized centrifugation, repeated filling, and evaporation for enrichment of drug solution or drugloaded nanoparticles in microcavities. ${ }^{66,67}$ Other ways for increasing the drug-loading capacity could be enlarging the size of the microneedle patch or developing new polymers with higher solubility or packing capacity of drug molecules. Second, the strategies which have been used for preparing sustained release microneedles often include intense physical/chemical procedures that may impact the structure or functionality of biotherapeutics, such as high temperature, use of organic solvent or UV light. New methods for minimizing the use of these intense conditions are still needed. Lastly, the effect of different release kinetics of the payloads from microneedle matrix on drug potency has not been systemically studied. Several studies have shown that by simply adjusting the components of the microneedle matrix or adjusting chemical modification level of the polymers, the release kinetics can be regulated and optimized. More indetailed mechanism and comparison studies are needed. 
Currently, there are dozens of clinical trial reports on investigating microneedle systems for treating diabetes, psoriatic plaques, topical anesthesia, and influenza vaccination. ${ }^{37,114,115}$ In most of these studies, commercially available hollow-microneedle systems were used while the clinical studies of polymeric microneedles are limited. ${ }^{116-120}$ Two phase I studies have investigated the piercing ability and safety of HA-based dissolving microneedles. The results showed that with the assistance of an applicator, the microneedles can be reproducibly penetrated into the skin with no obvious side effect. ${ }^{121,122}$ In another phase I clinical study, the safety and immunogenicity of HA-based dissolvable microneedles for delivery of H1N1, H3N2, and B seasonal influenza virus vaccine strains have been studied. The results showed that the designed microneedle patch is tolerable and can induce robust immune response. ${ }^{5}$ These studies revealed the potential for clinical translation of polymer microneedles. However, as far as we know, there are no clinical studies reported to date on the sustained release microneedles.

In conclusion, the microneedles with sustained release properties of drugs or vaccines have been successfully developed and applied for immune modulation and disease treatment in mice models. Many of them have shown superior potency than traditional fast-dissolving microneedles or free drug/vaccine formulations, revealing the potential of this strategy to be used as a patient-friendly replacement for conventional sustained release methods. Future success of this technology and clinical translation will rely on the combined efforts from researchers of engineering, pharmaceuticals, and immunology.

Conflict of Interest

None declared.

\section{Funding}

We acknowledge the financial support of the National Natural Science Foundation of China (No. 81961130395), Science and Technology Major Project of Sichuan Province (No. 2018SZDZX0018), and Sichuan Veterinary Medicine and Drug Innovation Group of China Agricultural Research System (CARS-SVDIP).

\section{References}

1 van der Maaden K, Jiskoot W, Bouwstra J. Microneedle technologies for (trans)dermal drug and vaccine delivery. J Control Release 2012;161(02):645-655

2 Kim YC, Park JH, Prausnitz MR. Microneedles for drug and vaccine delivery. Adv Drug Deliv Rev 2012;64(14):1547-1568

3 McAllister DV, Wang PM, Davis SP, et al. Microfabricated needles for transdermal delivery of macromolecules and nanoparticles: fabrication methods and transport studies. Proc Natl Acad Sci U S A 2003;100(24):13755-13760

4 Prausnitz MR, Langer R. Transdermal drug delivery. Nat Biotechnol 2008;26(11):1261-1268

5 Rouphael NG, Paine M, Mosley R, et al; TIV-MNP 2015 Study Group. The safety, immunogenicity, and acceptability of inactivated influenza vaccine delivered by microneedle patch (TIVMNP 2015): a randomised, partly blinded, placebo-controlled, phase 1 trial. Lancet 2017;390(10095);649-658

6 Chu LY, Ye L, Dong K, Compans RW, Yang C, Prausnitz MR. Enhanced stability of inactivated influenza vaccine encapsulated in dissolving microneedle patches. Pharm Res 2016;33(04): 868-878

7 Mistilis MJ, Bommarius AS, Prausnitz MR. Development of a thermostable microneedle patch for influenza vaccination. J Pharm Sci 2015;104(02):740-749

8 Schoellhammer CM, Blankschtein D, Langer R. Skin permeabilization for transdermal drug delivery: recent advances and future prospects. Expert Opin Drug Deliv 2014;11(03):393-407

9 Cevc G, Vierl U. Nanotechnology and the transdermal route: a state of the art review and critical appraisal. J Control Release 2010;141(03):277-299

10 Ito Y, Hagiwara E, Saeki A, Sugioka N, Takada K. Feasibility of microneedles for percutaneous absorption of insulin. Eur J Pharm Sci 2006;29(01):82-88

11 Lee JW, Choi SO, Felner EI, Prausnitz MR. Dissolving microneedle patch for transdermal delivery of human growth hormone. Small 2011;7(04):531-539

12 Indermun S, Luttge R, Choonara YE, et al. Current advances in the fabrication of microneedles for transdermal delivery. J Control Release 2014;185:130-138

13 Larrañeta E, McCrudden MT, Courtenay AJ, Donnelly RF. Microneedles: a new frontier in nanomedicine delivery. Pharm Res 2016;33(05):1055-1073

14 Tuan-Mahmood TM, McCrudden MT, Torrisi BM, et al. Microneedles for intradermal and transdermal drug delivery. Eur J Pharm Sci 2013;50(05):623-637

15 Sullivan SP, Murthy N, Prausnitz MR. Minimally invasive protein delivery with rapidly dissolving polymer microneedles. Adv Mater 2008;20(05):933-938

16 Park JH, Allen MG, Prausnitz MR. Biodegradable polymer microneedles: fabrication, mechanics and transdermal drug delivery. J Control Release 2005;104(01):51-66

17 Lee K, Lee CY, Jung H. Dissolving microneedles for transdermal drug administration prepared by stepwise controlled drawing of maltose. Biomaterials 2011;32(11):3134-3140

18 Lee JW, Park JH, Prausnitz MR. Dissolving microneedles for transdermal drug delivery. Biomaterials 2008;29(13):21132124

19 Lee JW, Han MR, Park JH. Polymer microneedles for transdermal drug delivery. J Drug Target 2013;21(03):211-223

20 Leone M, Mönkäre J, Bouwstra JA, Kersten G. Dissolving microneedle patches for dermal vaccination. Pharm Res 2017;34(11): 2223-2240

21 Herwadkar A, Banga AK. Peptide and protein transdermal drug delivery. Drug Discov Today Technol 2012;9(02):e71-e174

$22 \mathrm{Wu}$ F, Yang S, Yuan W, Jin T. Challenges and strategies in developing microneedle patches for transdermal delivery of protein and peptide therapeutics. Curr Pharm Biotechnol 2012;13(07):1292-1298

23 Matsuo K, Yokota Y, Zhai Y, et al. A low-invasive and effective transcutaneous immunization system using a novel dissolving microneedle array for soluble and particulate antigens. J Control Release 2012;161(01):10-17

24 Hiraishi Y, Nakagawa T, Quan YS, et al. Performance and characteristics evaluation of a sodium hyaluronate-based microneedle patch for a transcutaneous drug delivery system. Int J Pharm 2013;441(1-2):570-579

25 Guo L, Chen J, Qiu Y, Zhang S, Xu B, Gao Y. Enhanced transcutaneous immunization via dissolving microneedle array loaded with liposome encapsulated antigen and adjuvant. Int J Pharm 2013;447(1-2):22-30

26 Sullivan SP, Koutsonanos DG, Del Pilar Martin M, et al. Dissolving polymer microneedle patches for influenza vaccination. Nat Med 2010;16(08):915-920

27 Edens C, Dybdahl-Sissoko NC, Weldon WC, Oberste MS, Prausnitz MR. Inactivated polio vaccination using a microneedle patch is immunogenic in the rhesus macaque. Vaccine 2015;33(37): 4683-4690 
28 Edens C, Collins ML, Goodson JL, Rota PA, Prausnitz MR. A microneedle patch containing measles vaccine is immunogenic in non-human primates. Vaccine 2015;33(37):4712-4718

29 Vassilieva EV, Kalluri H, McAllister D, et al. Improved immunogenicity of individual influenza vaccine components delivered with a novel dissolving microneedle patch stable at room temperature. Drug Deliv Transl Res 2015;5(04):360-371

30 Quinn HL, Kearney MC, Courtenay AJ, McCrudden MT, Donnelly $\mathrm{RF}$. The role of microneedles for drug and vaccine delivery. Expert Opin Drug Deliv 2014;11(11):1769-1780

31 Prausnitz MR, Mikszta JA, Cormier M, Andrianov AK. Microneedlebased vaccines. In: Compans RW, Orenstein WA, eds. Vaccines for Pandemic Influenza. Berlin: Springer; 2009:369-393

32 Donnelly RF, Raj Singh TR, Woolfson AD. Microneedle-based drug delivery systems: microfabrication, drug delivery, and safety. Drug Deliv 2010;17(04):187-207

33 Li G, Badkar A, Nema S, Kolli CS, Banga AK. In vitro transdermal delivery of therapeutic antibodies using maltose microneedles. Int J Pharm 2009;368(1-2):109-115

34 Reichert JM, Rosensweig CJ, Faden LB, Dewitz MC. Monoclonal antibody successes in the clinic. Nat Biotechnol 2005;23(09): 1073-1078

35 Kamaly N, Yameen B, Wu J, Farokhzad OC. Degradable controlled-release polymers and polymeric nanoparticles: mechanisms of controlling drug release. Chem Rev 2016;116(04): 2602-2663

36 Hong X, Wei L, Wu F, et al. Dissolving and biodegradable microneedle technologies for transdermal sustained delivery of drug and vaccine. Drug Des Devel Ther 2013;7:945-952

37 Ye Y, Yu J, Wen D, Kahkoska AR, Gu Z. Polymeric microneedles for transdermal protein delivery. Adv Drug Deliv Rev 2018; 127:106-118

38 Chen MC, Huang SF, Lai KY, Ling MH. Fully embeddable chitosan microneedles as a sustained release depot for intradermal vaccination. Biomaterials 2013;34(12):3077-3086

39 Xie Y, Xu B, Gao Y. Controlled transdermal delivery of model drug compounds by MEMS microneedle array. Nanomedicine 2005;1 (02):184-190

40 Chu LY, Prausnitz MR. Separable arrowhead microneedles. J Control Release 2011;149(03):242-249

41 Lee K, Jung H. Drawing lithography for microneedles: a review of fundamentals and biomedical applications. Biomaterials 2012; 33(30):7309-7326

42 Moga KA, Bickford LR, Geil RD, et al. Rapidly-dissolvable microneedle patches via a highly scalable and reproducible soft lithography approach. Adv Mater 2013;25(36):5060-5066

43 Kim JD, Kim M, Yang H, Lee K, Jung H. Droplet-born air blowing: novel dissolving microneedle fabrication. J Control Release 2013; 170(03):430-436

44 Wang W, Nema S, Teagarden D. Protein aggregation-pathways and influencing factors. Int J Pharm 2010;390(02):89-99

45 Ratanji KD, Derrick JP, Dearman RJ, Kimber I. Immunogenicity of therapeutic proteins: influence of aggregation. J Immunotoxicol 2014;11(02):99-109

46 Zhu G, Mallery SR, Schwendeman SP. Stabilization of proteins encapsulated in injectable poly (lactide-co-glycolide). Nat Biotechnol 2000;18(01):52-57

47 Rothe A, Power BE, Hudson PJ. Therapeutic advances in rheumatology with the use of recombinant proteins. Nat Clin Pract Rheumatol 2008;4(11):605-614

48 Vora LK, Donnelly RF, Larrañeta E, González-Vázquez P, Thakur RRS, Vavia PR. Novel bilayer dissolving microneedle arrays with concentrated PLGA nano-microparticles for targeted intradermal delivery: proof of concept. J Control Release 2017; 265:93-101

$49 \mathrm{Ke} \mathrm{CJ}$, Lin YJ, Hu YC, et al. Multidrug release based on microneedle arrays filled with pH-responsive PLGA hollow microspheres. Biomaterials 2012;33(20):5156-5165
50 Park JH, Allen MG, Prausnitz MR. Polymer microneedles for controlled-release drug delivery. Pharm Res 2006;23(05): 1008-1019

51 Palakurthi NK, Correa ZM, Augsburger JJ, Banerjee RK. Toxicity of a biodegradable microneedle implant loaded with methotrexate as a sustained release device in normal rabbit eye: a pilot study. J Ocul Pharmacol Ther 2011;27(02):151-156

52 Kim M, Jung B, Park JH. Hydrogel swelling as a trigger to release biodegradable polymer microneedles in skin. Biomaterials 2012; 33(02):668-678

53 Demuth PC, Garcia-Beltran WF, Ai-Ling ML, Hammond PT, Irvine DJ. Composite dissolving microneedles for coordinated control of antigen and adjuvant delivery kinetics in transcutaneous vaccination. Adv Funct Mater 2013;23(02):161-172

54 Li W, Terry RN, Tang J, Feng MR, Schwendeman SP, Prausnitz MR. Rapidly separable microneedle patch for the sustained release of a contraceptive. Nat Biomed Eng 2019;3(03):220-229

55 Donnelly RF, Morrow DI, Singh TR, et al. Processing difficulties and instability of carbohydrate microneedle arrays. Drug Dev Ind Pharm 2009;35(10):1242-1254

56 DeMuth PC, Min Y, Irvine DJ, Hammond PT. Implantable silk composite microneedles for programmable vaccine release kinetics and enhanced immunogenicity in transcutaneous immunization. Adv Healthc Mater 2014;3(01):47-58

57 Wang C, Ye Y, Hochu GM, Sadeghifar H, Gu Z. Enhanced cancer immunotherapy by microneedle patch-assisted delivery of antiPD1 antibody. Nano Lett 2016;16(04):2334-2340

58 Chen BZ, Ashfaq M, Zhu DD, Zhang XP, Guo XD. Controlled delivery of insulin using rapidly separating microneedles fabricated from genipin-crosslinked gelatin. Macromol Rapid Commun 2018;39(20):e1800075

59 Lee J, Park SH, Seo IH, Lee KJ, Ryu W. Rapid and repeatable fabrication of high $\mathrm{A} / \mathrm{R}$ silk fibroin microneedles using thermally-drawn micromolds. Eur J Pharm Biopharm 2015;94:11-19

60 Tsioris K, Raja WK, Pritchard EM, et al. Fabrication of silk microneedles for controlled-release drug delivery. Adv Funct Mater 2012;22(02):330-335

61 Ye Y, Wang C, Zhang X, et al. A melanin-mediated cancer immunotherapy patch. Sci Immunol 2017;2(17):eaan5692

62 DeMuth PC, Moon JJ, Suh H, Hammond PT, Irvine DJ. Releasable layer-by-layer assembly of stabilized lipid nanocapsules on microneedles for enhanced transcutaneous vaccine delivery. ACS Nano 2012;6(09):8041-8051

63 Di J, Yao S, Ye Y, et al. Stretch-triggered drug delivery from wearable elastomer films containing therapeutic depots. ACS Nano 2015;9(09):9407-9415

$64 \mathrm{Ye} \mathrm{Y,} \mathrm{Yu} \mathrm{J,} \mathrm{Wang} \mathrm{C,} \mathrm{et} \mathrm{al.} \mathrm{Microneedles} \mathrm{integrated} \mathrm{with} \mathrm{pancreatic}$ cells and synthetic glucose-signal amplifiers for smart insulin delivery. Adv Mater 2016;28(16):3115-3121

65 Ye Y, Wang J, Hu Q et al. Synergistic transcutaneous immunotherapy enhances antitumor immune responses through delivery of checkpoint inhibitors. ACS Nano 2016;10(09):8956-8963

66 Wang J, Ye Y, Yu J, et al. Core-shell microneedle gel for selfregulated insulin delivery. ACS Nano 2018;12(03):2466-2473

67 Chen W, Tian R, Xu C, et al. Microneedle-array patches loaded with dual mineralized protein/peptide particles for type 2 diabetes therapy. Nat Commun 2017;8(01):1777

68 Koh LD, Cheng Y, Teng CP, et al. Structures, mechanical properties and applications of silk fibroin materials. Prog Polym Sci 2015;46:86-110

69 Liu Q Liu H, Fan Y. Preparation of silk fibroin carriers for controlled release. Microsc Res Tech 2017;80(03):312-320

70 You X, Chang JH, Ju BK, et al. Rapidly dissolving fibroin microneedles for transdermal drug delivery. Mater Sci Eng C 2011;31 (08):1632-1636

71 Jin HJ, Karageorgiou JPV, Kim UJ, et al. Water-stable silk films with reduced $\beta$-sheet content. Adv Funct Mater 2005;15(08): $1241-1247$ 
72 Lu Q, Hu X, Wang X, et al. Water-insoluble silk films with silk I structure. Acta Biomater 2010;6(04):1380-1387

73 Hofmann S, Foo CT, Rossetti F, et al. Silk fibroin as an organic polymer for controlled drug delivery. J Control Release 2006;111 (1-2):219-227

74 Yu J, Zhang Y, Kahkoska AR, Gu Z. Bioresponsive transcutaneous patches. Curr Opin Biotechnol 2017;48:28-32

75 Chen G, Yu J, Gu Z. Glucose-responsive microneedle patches for diabetes treatment. J Diabetes Sci Technol 2019;13(01):41-48

76 Mahapatro A, Singh DK. Biodegradable nanoparticles are excellent vehicle for site directed in-vivo delivery of drugs and vaccines. J Nanobiotechnology 2011;9:55

77 Taghizadeh B, Taranejoo S, Monemian SA, et al. Classification of stimuli-responsive polymers as anticancer drug delivery systems. Drug Deliv 2015;22(02):145-155

78 Mura S, Nicolas J, Couvreur P. Stimuli-responsive nanocarriers for drug delivery. Nat Mater 2013;12(11):991-1003

$79 \mathrm{Yu}$ J, Zhang Y, Ye Y, et al. Microneedle-array patches loaded with hypoxia-sensitive vesicles provide fast glucose-responsive insulin delivery. Proc Natl Acad Sci U S A 2015;112(27):8260-8265

80 Zhang Y, Wang J, Yu J, et al. Bioresponsive microneedles with a sheath structure for h2 o2 and ph cascade-triggered insulin delivery. Small 2018;14(14):e1704181

81 Romani N, Flacher V, Tripp CH, Sparber F, Ebner S, Stoitzner P. Targeting skin dendritic cells to improve intradermal vaccination. Curr Top Microbiol Immunol 2012;351:113-138

82 Sparber F, Tripp CH, Hermann M, Romani N, Stoitzner P. Langerhans cells and dermal dendritic cells capture protein antigens in the skin: possible targets for vaccination through the skin. Immunobiology 2010;215(9-10):770-779

83 Kim YC, Jarrahian C, Zehrung D, Mitragotri S, Prausnitz MR. Delivery systems for intradermal vaccination. Curr Top Microbiol Immunol 2012;351:77-112

84 Segura E, Villadangos JA. Antigen presentation by dendritic cells in vivo. Curr Opin Immunol 2009;21(01):105-110

85 Zaba LC, Krueger JG, Lowes MA. Resident and "inflammatory" dendritic cells in human skin. J Invest Dermatol 2009;129(02): 302-308

86 Quan FS, Kim YC, Compans RW, Prausnitz MR, Kang SM. Dose sparing enabled by skin immunization with influenza virus-like particle vaccine using microneedles. J Control Release 2010;147 (03):326-332

87 Song JM, Kim YC, O E, Compans RW, Prausnitz MR, Kang SM. DNA vaccination in the skin using microneedles improves protection against influenza. Mol Ther 2012;20(07):1472-1480

88 Glenn GM, Taylor DN, Li X, Frankel S, Montemarano A, Alving CR. Transcutaneous immunization: a human vaccine delivery strategy using a patch. Nat Med 2000;6(12):1403-1406

89 Mikszta JA, Alarcon JB, Brittingham JM, Sutter DE, Pettis RJ, Harvey NG. Improved genetic immunization via micromechanical disruption of skin-barrier function and targeted epidermal delivery. Nat Med 2002;8(04):415-419

90 Kim YC, Quan FS, Yoo DG, Compans RW, Kang SM, Prausnitz MR. Improved influenza vaccination in the skin using vaccine coated microneedles. Vaccine 2009;27(49):6932-6938

91 Kupper TS, Fuhlbrigge RC. Immune surveillance in the skin: mechanisms and clinical consequences. Nat Rev Immunol 2004;4(03):211-222

92 Lin WH, Kouyos RD, Adams RJ, Grenfell BT, Griffin DE. Prolonged persistence of measles virus RNA is characteristic of primary infection dynamics. Proc Natl Acad Sci U S A 2012;109(37): 14989-14994

93 Simon ID, Publicover J, Rose JK. Replication and propagation of attenuated vesicular stomatitis virus vectors in vivo: vector spread correlates with induction of immune responses and persistence of genomic RNA. J Virol 2007;81(04):2078-2082

94 Tam HH, Melo MB, Kang M, et al. Sustained antigen availability during germinal center initiation enhances antibody responses to vaccination. Proc Natl Acad Sci U S A 2016;113(43): E6639-E6648

95 Zhao L, Seth A, Wibowo N, et al. Nanoparticle vaccines. Vaccine 2014;32(03):327-337

96 Demento SL, Cui W, Criscione JM, et al. Role of sustained antigen release from nanoparticle vaccines in shaping the T cell memory phenotype. Biomaterials 2012;33(19):4957-4964

97 Moon JJ, Suh H, Li AV, Ockenhouse CF, Yadava A, Irvine DJ. Enhancing humoral responses to a malaria antigen with nanoparticle vaccines that expand Tfh cells and promote germinal center induction. Proc Natl Acad Sci U S A 2012;109(04): 1080-1085

98 Lu S. Heterologous prime-boost vaccination. Curr Opin Immunol 2009;21(03):346-351

99 Arya J, Prausnitz MR. Microneedle patches for vaccination in developing countries. J Control Release 2016;240:135-141

100 Wong KK, Li WA, Mooney DJ, Dranoff G. Advances in therapeutic cancer vaccines. Adv Immunol 2016;130:191-249

101 Tumeh PC, Harview CL, Yearley JH, et al. PD-1 blockade induces responses by inhibiting adaptive immune resistance. Nature 2014;515(7528):568-571

102 Banchereau J, Palucka K. Immunotherapy: cancer vaccines on the move. Nat Rev Clin Oncol 2018;15(01):9-10

103 Xia AL, Xu Y, Lu XJ. Cancer immunotherapy: challenges and clinical applications. J Med Genet 2019;56(01):1-3

104 Rappuoli R, Pizza M, Del Giudice G, De Gregorio E. Vaccines, new opportunities for a new society. Proc Natl Acad Sci U S A 2014; 111(34):12288-12293

105 Sharma P, Allison JP. The future of immune checkpoint therapy. Science 2015;348(6230):56-61

106 Skyler JS. Diabetes mellitus: pathogenesis and treatment strategies. J Med Chem 2004;47(17):4113-4117

107 Chen H, Zhu H, Zheng J, et al. Iontophoresis-driven penetration of nanovesicles through microneedle-induced skin microchannels for enhancing transdermal delivery of insulin. J Control Release 2009;139(01):63-72

108 Wu Y, Gao Y, Qin G, et al. Sustained release of insulin through skin by intradermal microdelivery system. Biomed Microdevices 2010;12(04):665-671

109 Lee IC, Lin WM, Shu JC, Tsai SW, Chen CH, Tsai MT. Formulation of two-layer dissolving polymeric microneedle patches for insulin transdermal delivery in diabetic mice. J Biomed Mater Res A 2017;105(01):84-93

110 Weinger K, Jacobson AM, Draelos MT, Finkelstein DM, Simonson DC. Blood glucose estimation and symptoms during hyperglycemia and hypoglycemia in patients with insulin-dependent diabetes mellitus. Am J Med 1995;98(01):22-31

111 Dagogo-Jack SE, Craft S, Cryer PE. Hypoglycemia-associated autonomic failure in insulin-dependent diabetes mellitus. Recent antecedent hypoglycemia reduces autonomic responses to, symptoms of, and defense against subsequent hypoglycemia. J Clin Invest 1993;91(03):819-828

112 Wilinska ME, Budiman ES, Taub MB, et al. Overnight closed-loop insulin delivery with model predictive control: assessment of hypoglycemia and hyperglycemia risk using simulation studies. J Diabetes Sci Technol 2009;3(05):1109-1120

113 Seong KY, Seo MS, Hwang DY, et al. A self-adherent, bulletshaped microneedle patch for controlled transdermal delivery of insulin. J Control Release 2017;265:48-56

114 Pettis RJ, Harvey AJ. Microneedle delivery: clinical studies and emerging medical applications. Ther Deliv 2012;3(03):357-371

115 Bhatnagar S, Dave K, Venuganti VVK. Microneedles in the clinic. J Control Release 2017;260:164-182

116 Norman JJ, Brown MR, Raviele NA, Prausnitz MR, Felner EI. Faster pharmacokinetics and increased patient acceptance of intradermal insulin delivery using a single hollow microneedle in children and adolescents with type 1 diabetes. Pediatr Diabetes 2013;14(06):459-465 
117 Marshall S, Sahm LJ, Moore AC. Microneedle technology for immunisation: perception, acceptability and suitability for paediatric use. Vaccine 2016;34(06):723-734

118 Nguyen TT, Park JH. Human studies with microneedles for evaluation of their efficacy and safety. Expert Opin Drug Deliv 2018;15(03):235-245

119 Kochba E, Levin Y, Raz I, Cahn A. Improved insulin pharmacokinetics using a novel microneedle device for intradermal delivery in patients with type 2 diabetes. Diabetes Technol Ther 2016;18 (09):525-531
120 Gupta J, Felner EI, Prausnitz MR. Minimally invasive insulin delivery in subjects with type 1 diabetes using hollow microneedles. Diabetes Technol Ther 2009;11(06):329-337

121 Hirobe S, Azukizawa H, Matsuo K, et al. Development and clinical study of a self-dissolving microneedle patch for transcutaneous immunization device. Pharm Res 2013;30(10):2664-2674

122 Hirobe S, Azukizawa H, Hanafusa T, et al. Clinical study and stability assessment of a novel transcutaneous influenza vaccination using a dissolving microneedle patch. Biomaterials 2015; $57: 50-58$ 